\title{
The Influence of Placement Time of Blood Samples on the Accuracy of Blood Glucose Measurement
}

\author{
Xiaoyang Zhao ${ }^{1}$, Jiasheng Huang ${ }^{1}$, Mingjiang Gong ${ }^{2}$ \\ ${ }^{1}$ The Outpatient Department of CAFP Institute of Politics, Shanghai, China \\ ${ }^{2}$ Shanghai Dian Medical Laboratory Limited, Shanghai, China \\ Email: zhao.xiaoyang@hotmail.com
}

How to cite this paper: Zhao, X.Y., Huang, J.S. and Gong, M.J. (2018) The Influence of Placement Time of Blood Samples on the Accuracy of Blood Glucose Measurement. Health, 10, 1090-1094. https://doi.org/10.4236/health.2018.108082

Received: July 2, 2018

Accepted: August 24, 2018

Published: August 27, 2018

Copyright (c) 2018 by authors and Scientific Research Publishing Inc. This work is licensed under the Creative Commons Attribution International License (CC BY 4.0).

http://creativecommons.org/licenses/by/4.0/

\begin{abstract}
This study aims to explore the influence brought by the decrease degree in blood glucose during the period when diabetes patients' blood samples are collected through fasting blood and sent to the laboratory for detection on the accuracy on blood glucose measurement. Methods: This study is conducted by detecting fasting blood glucose of 150 patients who came to our hospital for physical examination and collecting their general information. Blood glucose concentration is measured through centrifugation after blood samples are placed for $0 \mathrm{~min}, 60 \mathrm{~min}, 120 \mathrm{~min}$ and $180 \mathrm{~min}$.
\end{abstract}

\section{Keywords}

Fasting Blood, Blood Glucose Detection, Time of Inspection, Detection Result, Influence

\section{Introduction}

With the continuous improvement of people's living standards and acceleration of the aging process, the morbidity of diabetes has continued to rise. Worse still, suffering from hyperglycemia for a long time can lead to dysfunction of multiple organs, which seriously affects people's life and health. Therefore, the accuracy of monitoring blood detection results will directly affect treatment and medication of patients in the clinical treatment course [1]. The fasting blood of patients in wards is usually done by night nurses, which is usually completed at about 6.am. However, after collecting the blood samples, these nurses fail to do special treatment, but place them under room temperature, and send them to the labor- 
atory for inspection during the work hours. The laboratory usually starts working at 8:00 am and all blood samples must be collected for pre-processing due to the large number of inpatients, which means such samples are detected almost by 9:00 am [2]. Therefore, during the time when blood samples are collected by fasting blood and sent to laboratory for detection, the concentration of blood samples may decrease due to the consumption of blood glucose caused by the respiration of red blood cells, thus influencing the accuracy of fasting blood glucose measurement of diabetics as well as controlling blood glucose and clinical medication of diabetics.

\section{Objects and Methods}

1) The research subjects are 150 patients who come to the outpatient department of our hospital for physical examination, including 117 males and $33 \mathrm{fe}-$ males aged from 24 to 83 , with an average age of 50.9 years old.

2) The detecting instruments and reagents include Mindray BS-420 automatic biochemical analyzer, the glucose measuring kit (through the oxidase method), quality control products and calibrators provided by Mindray as well as blood collection tubes provided by Zhejiang Kangshi Medical Devices Co., Ltd.

3) As for the detection method, venous blood from those came for physical examination were collected in the early morning through fasting blood. Blood collected from each objective shall be divided into 4 test tubes, of which each contained $2 \mathrm{ml}$, was respectively numbered tube A, B, C and D and immediately sent to the laboratory for detection. Under room temperature, the serum extraction of a total of 4 blood samples from each research subject is made through centrifugation at different time points of $0 \mathrm{~min}, 60 \mathrm{~min}, 120 \mathrm{~min}$ and $180 \mathrm{~min}$, and the blood glucose concentration of each blood sample at different time points is recorded in time. If the blood glucose detection results are higher than $6 \mathrm{mmol} / \mathrm{l}$ which is impaired glucose tolerance, it should be deemed as hypoglycemia and hyperglycemia otherwise.

4) Statistical Processing. In the course of results processing of this study, statistical software SPSS was adopted for statistical data processing, where $\mathrm{x}- \pm \mathrm{s}$ indicates the measurement data of the normal distribution. After data processing, it is found that when $\mathrm{P}<0.05$, the data collected is statistically significant.

\section{Results}

1) The 150 whole blood samples are divided into hypoglycemia group $(n=81)$ and hyperglycemia group $(\mathrm{n}=69)$ and the blood glucose detecting results through centrifugation at $0 \mathrm{~min}, 60 \mathrm{~min}, 120 \mathrm{~min}$ and $180 \mathrm{~min}$ under room temperature are shown in Table $1 . \mathrm{P}<0.05$ can be drawn in the difference in time between the hypoglycemia and hyperglycemia blood glucose groups. Therefore, there is a significant difference in time between the hyperglycemia group and the hypoglycemia group, as shown in Table 2. 
Table 1. Results of blood glucose concentration at different time points ( $\mathrm{mmol} / \mathrm{l})$.

\begin{tabular}{ccc}
\hline Time of Placement & Hypoglycemia Group & Hyperglycemia Group \\
\hline $0 \mathrm{~min}$ & $5.5116 \pm 0.125$ & $6.02 \pm 0.23646$ \\
$60 \mathrm{~min}$ & $5.102 \pm 0.206$ & $4.74 \pm 0.22511$ \\
$120 \mathrm{~min}$ & $4.75 \pm 0.206$ & $4.71 \pm 0.22754$ \\
$180 \mathrm{~min}$ & $4.4246 \pm 0.215$ & $4 \pm 0.23121$ \\
\hline
\end{tabular}

Table 2. Comparison of differences in time between hypoglycemia group and hyperglycemia group.

\begin{tabular}{|c|c|c|c|}
\hline Time of Placement & & T-value & P-value \\
\hline \multirow[t]{2}{*}{$0 \mathrm{~min}$} & With assumption of Homogeneity of variance & 8.845 & $<0.05$ \\
\hline & $\begin{array}{l}\text { Without assumption of Homogeneity of } \\
\text { variance }\end{array}$ & 8.200 & $<0.05$ \\
\hline \multirow[t]{2}{*}{$60 \mathrm{~min}$} & With assumption of Homogeneity of variance & 8.313 & $<0.05$ \\
\hline & $\begin{array}{l}\text { Without assumption of Homogeneity of } \\
\text { variance }\end{array}$ & 7.737 & $<0.05$ \\
\hline \multirow[t]{2}{*}{$120 \mathrm{~min}$} & With assumption of Homogeneity of variance & 8.337 & $<0.05$ \\
\hline & $\begin{array}{l}\text { Without assumption of Homogeneity of } \\
\text { variance }\end{array}$ & 7.758 & $<0.05$ \\
\hline \multirow[t]{2}{*}{$180 \mathrm{~min}$} & With assumption of Homogeneity of variance & 8.072 & $<0.05$ \\
\hline & $\begin{array}{l}\text { Without assumption of Homogeneity of } \\
\text { variance }\end{array}$ & 7.512 & $<0.05$ \\
\hline
\end{tabular}

Note: $\mathrm{P}<0.05$, with statistical significance.

2) It can be found that there is significant change in the blood glucose concentration of blood samples in both Hyperglycemia group and hypoglycemia group after being placed for $0 \mathrm{~min}, 60 \mathrm{~min}, 120 \mathrm{~min}$ and $180 \mathrm{~min}$ at room temperature, with significant differences among specimens and different time points, namely, $\mathrm{P}<0.05$, with statistical significance. On the other hand, from the difference mean and standard deviation, it can be seen that the biggest decrease in blood glucose concentration occurs at $60 \mathrm{~min}$ in both hyperglycemia group and hypoglycemia group, as shown in Table 3 and Table 4.

\section{Conclusion}

It can be seen in this experiment that blood glucose of blood samples from both normal people and diabetics decrease rapidly after such samples are collected, especially in diabetic patients, which is most significant at the time point $60 \mathrm{mi}-$ nutes. Therefore, the centrifugation detection shall be conducted in a timely manner within one hour after the blood samples are collected. Otherwise, blood cells can still react with serum for reaction, which may cause decrease in blood glucose concentration. Conditions not permitting immediate detection, centrifuged blood samples should also be placed in a $4^{\circ} \mathrm{C}$ freezer for storage. 
Table 3. Comparison of blood glucose concentrations of hypoglycemia group in different time periods.

\begin{tabular}{ccccc}
\hline Time Matching & $\begin{array}{c}\text { Mean Difference in } \\
\text { Matching (E) }\end{array}$ & Standard Deviation & T-value & P-value \\
\hline 0 min - 60 min & 0.40963 & 0.37296 & 9.885 & $<0.05$ \\
60 min - 120 min & 0.35198 & 0.33877 & 9.351 & $<0.05$ \\
120 min - 180 min & 0.32543 & 0.25476 & 11.497 & $<0.05$ \\
\hline
\end{tabular}

Note: $\mathrm{P}<0.05$, with statistical significance.

Table 4. Comparison of blood glucose concentrations of hyperglycemia group in different time periods.

\begin{tabular}{ccccc}
\hline & & & \\
Time Matching & $\begin{array}{c}\text { Mean Difference in } \\
\text { Matching (E) }\end{array}$ & $\begin{array}{c}\text { Standard Devia- } \\
\text { tion }\end{array}$ & T-value & P-value \\
\hline 0 min - 60 min & 0.59029 & 0.37653 & 13.022 & $<0.05$ \\
60 min - 120 min & 0.32884 & 0.28865 & 9.463 & $<0.05$ \\
120 min - 180 min & 0.35391 & 0.32222 & 9.124 & $<0.05$ \\
\hline
\end{tabular}

Note: $\mathrm{P}<0.05$, with statistical significance.

\section{Discussion}

Saccharides come with many important biological functions in the human body, which are both the main source of energy needed by the body and an important part of nutrients for organism.

Blood glucose refers to saccharides in the blood of human, mostly glucose. More than $70 \%$ of energy in histocyte of human body is given by oxidation of glucose, which shows the importance of blood glucose balance to organism. [3] After the blood is isolated from human body, the glucose can be decomposed due to the degradation of leukocytes, respiration of red blood cells, bacterial contamination and other factors, resulting in a decrease in blood glucose concentration.

Diabetes has been the third most serious chronic non-communicable disease after tumors and cardiovascular and cerebrovascular diseases as well as one of the leading causes of death and disability. China has become the country home to the world's largest diabetes patients and there are more than 150 million people with pre-diabetes [4]. Therefore, blood glucose detection is of great significance in clinical diagnosis and treatment of diabetes. In order to improve the accuracy of blood glucose detection, prevent glycolysis of blood glucose before detection and provide relatively objective and accurate blood glucose results for clinical treatment, it is necessary to quickly detect blood samples in laboratory and make proper pre-processing of blood samples with collaboration with clinicians.

\section{Conflicts of Interest}

The authors declare no conflicts of interest regarding the publication of this paper. 


\section{References}

[1] Niu, Z.B. and Li, X.J. (2014) A Comparative Study on the Verification of Accuracy of Blood Glucose Meters Based on Different Principles. Journal of Hebei Medicine, $20,1402$.

[2] Wang, Y.J., Zhang, X.L., Wang, B.H., Zhang, J. and Bi, X.H. (2011) The Effect of Different Placement Time of Blood Samples on the Results of 17 Biochemical Tests. Medical Journal of the Chinese People Armed Police Forces, 22, 1034.

[3] Liu, X.Z. (2012) The Effect of Placement Time and Treatment of Blood Samples on Blood Glucose Concentration. International Journal of Laboratory Medicine, 33, 1871.

[4] Wang, J.H. (2007) The Hazards of Diabetes. King Reader (Doctor \& Pharmacy), No. $1,13$. 